\title{
Research on Tactical Mode Analysis of Sports Video
}

\author{
Gang Liu, Yang Wang and Jing-meng Sun \\ Physical Education Department, Harbin Engineering University, Harbin 150000, \\ China \\ Liuganglg2014@126.com
}

\begin{abstract}
A new tactic analysis of broadcast sports video is proposed based on player trojectory information. Tactic analysis of sports video aims to recognize and discover tactic modes and match strategies that teams and personal players used in the games. Based on players and ball trajectory. First presents an interactive relationship analysis of tentporal and spatial algorithm based on time fragment slice. According to the speed and distance of trajectory shape and the distance measurement and each trajectory in time fragment, to use graphical model was constructed to express tactical video gane sports event. Through the analysis of each component fragment of interaction trajectory, the offensive incidents of tactical mode carried out hierarchical recognition of coarse tofine in football game video. In the coarse recognition process, the interaction mode is divided into a coordinated attack and personal attack. In fine recognition further, the coordinated attack mode is divided into interception attack and no interception attack. The personal attack mode is divided into direct attack and the dribbling-attack.
\end{abstract}

Keywords: Tactic analysis, Trajectory, Tâctieal mode Sports video

\section{Introduction}

Traditional sports video analysis is made to ordinary audience [1], mainly referring to detection and recognition of wonderful shots and competitive events and acquisition of video fragments which are interesting to watchers, which is called semantic analysis of video contents [2-3]. In recent years, for the proposal and further practice of the concept of computer aided training of sports events, along with awareness of the importance of tactical information to understand and enjoy such contests, the tactical mode recognition and analysis of related videos have become a new hot concern in the sports video analysis field, which we name tactical analysis of video contents [4].

The purpose of such analysis is to discover the tactical mode or competition strategy taken by personal players or members to complete one action or task in the competition. Take footbal game for instance. Professional players or full-time coaches expect to take advantage of computer's powerful calculating ability to recognize automatically pass and catch techniques when both teams are assaulting and defending in the game, so as to formulate according training plan and game strategy to improve actual combat capability. As far as its practical use is concerned, in order to obtain tactical analysis results relating to the game, sport professionals will take manual labeling means to finish it [5]. The work is considerably time-consuming, labor-intensive, and resource-intensive. As a result, researches and presentation of automatic tactical analysis methods are growing to a question for urgent settlement in the video analysis field [6]. 
The paper introduced a tactical mode analyzing approach which is used for broadcast football game videos. As the most representative team event, football game appeals tremendous fans in China and even the whole world. The game program has incomparably overwhelming audience groups than other competitive physical sports. At present, studies on tactical analysis methods for football game videos are quite limited. In the work [7], the author acquired visual sequences of the game through multiple static cameras, trajectory player in multi-look sequences. Based on multi-look geometrical relationship, he figured out true locations of players on the court. By introducing two tactical ideas: shortest moving time and player control area, he completed the evaluation of each party's tactical situation in the game. The paper [8] based on time-series data, raised a method which makes hierarchical classification of ball passing modes leading to gain scores in the football match. By analyzing the spatial distribution relationship of 22 players and ball trails in the competition, Kang [9] put forward a numerical calculation model for tactical assessment of football game. Throughout all current works, we can see that they experimented based on the data acquired through special means, e.g. special photographing device (multi-camera) conf guration. Manpower was used to make initial labeling of data. Data were generated th ough computer simulation. Thus, those methods face huge environmental challenges when being used in practice. To overcome the weakness, it proposes a tactical analysis method based on airing sport videos, used to recognize the tactical mode for offensive action in the football game. Broadcast video is the main form of television relaying spon competition, of abundant volume of data. Such video is more easily obtained than special videos which are acquired through special camera device in special environment. Therefore, the proposed method is practically more meaningful [10-11].

\section{Middle Expression Construction of Video Content Tactics}

\subsection{Mosaic Trajectory Calculation}

Mosaic trajectory calculation is based on transform the principle of affine video frames. By removing the broadcast video of the moving camera, from a video frame obtained trajectory image coordinates to transform into unified coordinate space. Here, we use the global motion estimation algorithm to complete the calculation of mosaic trajectory. Using homogeneous coordinates global motion estimation formula rewritten [12] as follows:

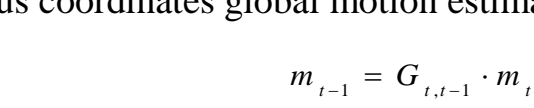

The $m_{t-1}$ represents atrary picture coordinates in $t-1$ frame, the corresponding point in the $t$ frame is $m$. The $G_{t, t-1}$ represents $t$ to $t-1$ frame transformation matrix of global motion estînation. For given video sequence $V=\left\{f_{1}, \ldots, f_{n}\right\}$, where, $f_{i}$ represents the $i$ frame in the sequence. A trajectory in sequence $V$ is $T=\left\{p_{1}, \ldots, p_{n}\right\}$, where $p_{i}$ represents trajectory $T$ position in the intra-frame $f_{i}$. According to the timing relationship of each frame in the sequence and Formula 1. You can calculate $p_{i}$ the mapping coordinates in the intraframe $f_{i}$. Let $p_{i}$ homogeneous coordinates is $P_{i}$, the mapping coordinates calculated is as shown in the following formula:

$$
P_{i}=\prod_{t=2}^{i} H_{t, t-1} \cdot P_{i}
$$


Let $P_{i}$ was under homogeneous coordinates $P_{i}$ mapping points in $f_{i}$ frame. Using formula (2) can be mapped trajectory $T$ of each frame into a unified position in the coordinate system.

After the treatment of mosaic trajectory is equivalent to using a fixed camera video results. To eliminate the camera motion video sequences generated in the inter frame displacement.

\subsection{Interactive Relationship Analysis of Temporal and Spatial}

The core idea of interactive trajectory is: Using a compact form of expression effectively describes events for the interaction relationship between the players and the ball during the attack incident. As mentioned before, in football game, the ball is the execution object of player completed tactical target. All tactical intention will eventually be completed by the control of the ball. Therefore, the players and the ball trajectory are tactical mode recognition and mainly information of analysis. In football game, the interactive relationship between the players and the ball can be divided into two kinds of typical model. Namely, passing and dribbling. In this paper, the method is proposed interactive relationship analysis of temporal and spatial based on players and the ball trajectory information. Select passing trajectory or dribbling trajectory from the mosaic trajectory in each time segment fragments, then the trajectory segments were spliced according to temporal relations. To form a new track to call interactive trajectory [13].

Figure 1 will give attack events to divide into several segments of different game period. The segmented game period can be classified into two categories: passing and dribbling period. Respectively corresponding to twotypical interaetive mode of the above analysis, for each game period time can be further divided into several fragments, every fragment of time to contain one or more smooth and eontinuous objece trajectories. For passing and dribbling period, using the deterministic method and probabilistic graphical model extraction method of mosaic trajectory corresponding to build interactive trajectory. The specific as shown in algorithm1.

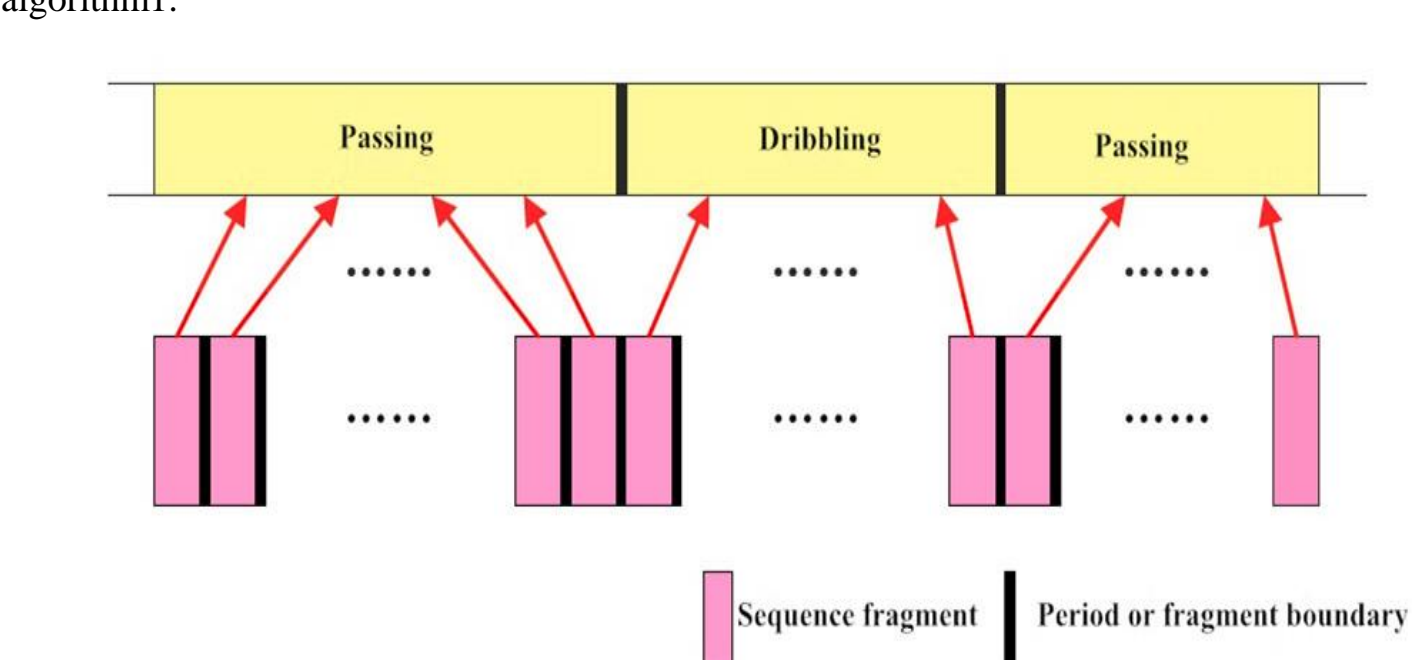

Figure 1. Period/interval Structure for Temporal-spatial Interaction Analysis

Algorithm1 Aggregate trajectory generation based on temporal-spatial interaction analysis Input: Mosaic trajectory set $M T=\left\{l_{b}(t), l_{p 1}(t), \ldots, l_{p n}(t)\right\}$ 
Output: Interactive trajectory $A T$

1. The mosaic trajectory set $M T$ of each path is Gauss smoothing to remove noise.

2. According to the equal interval $t$ will all mosaic trajectory to divide into T-subsets, which, $S_{k}=\left\{l_{b}^{k}(t), l_{p 1}^{k}(t), \ldots, l_{p n_{k}}^{k}(t)\right\}, l_{\#}^{k}(t)$ represents the $k$ trajectory sub-fragment of trajectory $l_{\#}(t)$ in $M T .1 \leq k \leq T$.

3. Passing and dribbling period using temporal similarity metrics method to select the part of the trajectory of each time interval sub-fragment of the trajectory is as interactive trajectory $A T$

Set up $k=1$

For $S_{k}$, calculate the similarity $F_{b, p_{j}}^{k}=D_{b, p_{j}}^{k} \cdot H_{b, p_{j}}^{k}$, which, $D_{b, p_{j}}^{k}$ and $H_{b, p_{j}}^{k}$ are trajectory fragment $l_{b}^{k}(t) l_{p j}^{k}(t)$ in space distance and shape measurement.

Passing period using deterministic methods to build sub-fragment of interactive trajectory

Dribbling period using probability graph model method to build sub-ragment of interactive trajectory

If $k<T$ then, repeat step3 else the execution step4

3. According to the time interval index $k$ will select al the trajectory fragment to connect interactive trajectory $A T$

\section{Recognition of Tactical Mode}

\subsection{The Coarse Recognition of Interactive Mode}

During coarse recognition, interactive patters include coordinated attack mode and personal attack mode. The forme one is a typeo of tactics by which a few players attack the other team through pass and catch in the competition. The latter one refers to the tactical purpose fulfilled by only one-player through dribbling or direct shooting.

Especially given attack eyent G, according to algorithm1, using the tactical Middle expression to construct algorithm to obtain interactive trajectory $A T=\left\{s_{1}, \ldots, s_{n}\right\}$, where, $s_{i}$ represents the $i$ trajectory fragment in the interactive trajectory. It can be defined as a coarse classification Standard as folloys:

$$
C(G)=\sum_{i=1}^{n} \operatorname{ball}\left(s_{i}\right)
$$

Where, the function ball(.) is as the indicator function:

$$
\begin{aligned}
& \operatorname{ball}(x)= \begin{cases}1 & \mathrm{X} \text { is the ball trajectory fragment } \\
0 & \mathrm{X} \text { is not the ball trajectory fragment }\end{cases} \\
& \text { Coarse classification model is defined as: }
\end{aligned}
$$

$$
P_{\text {coarse }}(G)= \begin{cases}\text { Coordinated attack } & C_{\text {coarse }}(G)>0 \\ \text { Personal attack } & C_{\text {coarse }}(G)=0\end{cases}
$$

\subsection{Fine Recognition of Interactive Mode}

In the fine recognition, coordinated attack and personal attack are further subdivided into four types of modes as in table1: for the former mode, it has interception attack and noninterception attack whether balls are tackled by defensive team's players in the attack 
progress; for the latter mode, it includes direct attack and dribbling attack whether players dribble balls in the attack process.

3.2.1. Fine Recognition of Coordinated Attack Mode. Set the interactive trail of recognized coordinated attack event AT, and remove all fragments about ball's trajectory, then we can have the subset $S A T=\left\{s p_{1}, \ldots, s p_{m}\right\}$ which only include fragments only about player trail. Now we can define the recognition standard of coordinated attack fine recognition as:

$$
C_{\text {fine }_{-}}(G)=\sum_{i=2}^{m}\left\{1-\delta\left[\text { player }\left(s p_{i}-\operatorname{player}\left(s p_{i-1}\right)\right]\right\}\right.
$$

Which, $\delta$ is Kroneckerdate function. player $(x)$ is used to identify team of the trajectory fragment $x$. Therefore, fine classification of cooperative attack can be completêd in accordance with the following formula:

$$
P_{\text {fine }-c}(G)=\left\{\begin{array}{l}
\text { Interception attack } \\
\text { No interception attack }
\end{array}\right.
$$$$
\begin{aligned}
& C_{\text {fine } c}(G)>0 \\
& C_{\text {fine }}(G)=0
\end{aligned}
$$

Table 1. Description of Tactic Interaction Modes

\begin{tabular}{|c|c|c|}
\hline Coarse recognition & Fine recognition & Description \\
\hline Coordinated attack & Interception attack & $\begin{array}{c}\text { The ball is intercepted by one } \\
\text { or more defenders in the } \\
\text { offensive }\end{array}$ \\
\cline { 2 - 4 } & No interception attack & $\begin{array}{c}\text { The ball is not intercepted by } \\
\text { defenders to In the offensive }\end{array}$ \\
\hline Personal attack & $\begin{array}{c}\text { The players are not ball } \\
\text { dribbling in the offensive }\end{array}$ \\
\hline
\end{tabular}

3.2.2. Fine Recognition of Personal Attack Mode. In the personal attack mode, direct attack involves mainly penalty shootout Tike free kick, place kick and the like. In contrast, dribbling attack means one player completing attack or shoot through ball dribbling to shun from the interception by the defender the opposite team. The sophisticated classification of personal attack mode is realized by the hypothesis testing method for the trail space analysis.

By analyzing trajectory spatial distribution in the personal attack mode, for direct attack, the trail is linearly distributed in three-dimensional and two-dimensional space as seen in Figure 2-3; for dribbling attack, the path is not linearly distributed in both 2D and 3D space (Figure 4\&5). Such distribution can be validated through analysis of completing process in both modes. When foul shot is implemented, players will run quickly towards the ball for a short distance complete the shot action. That's why the trail is approximately of linear distribution in the space. For dribbling attack, the player should try to shun from being intervened by defending members when he is carrying the ball. Therefore the attacking route is of curvilinear spatial distribution. With trail spatial distribution, we can complete refined classification of personal attack by Hypothesis test method [14]. 
International Journal of Multimedia and Ubiquitous Engineering Vol. 9, No. 12 (2014)

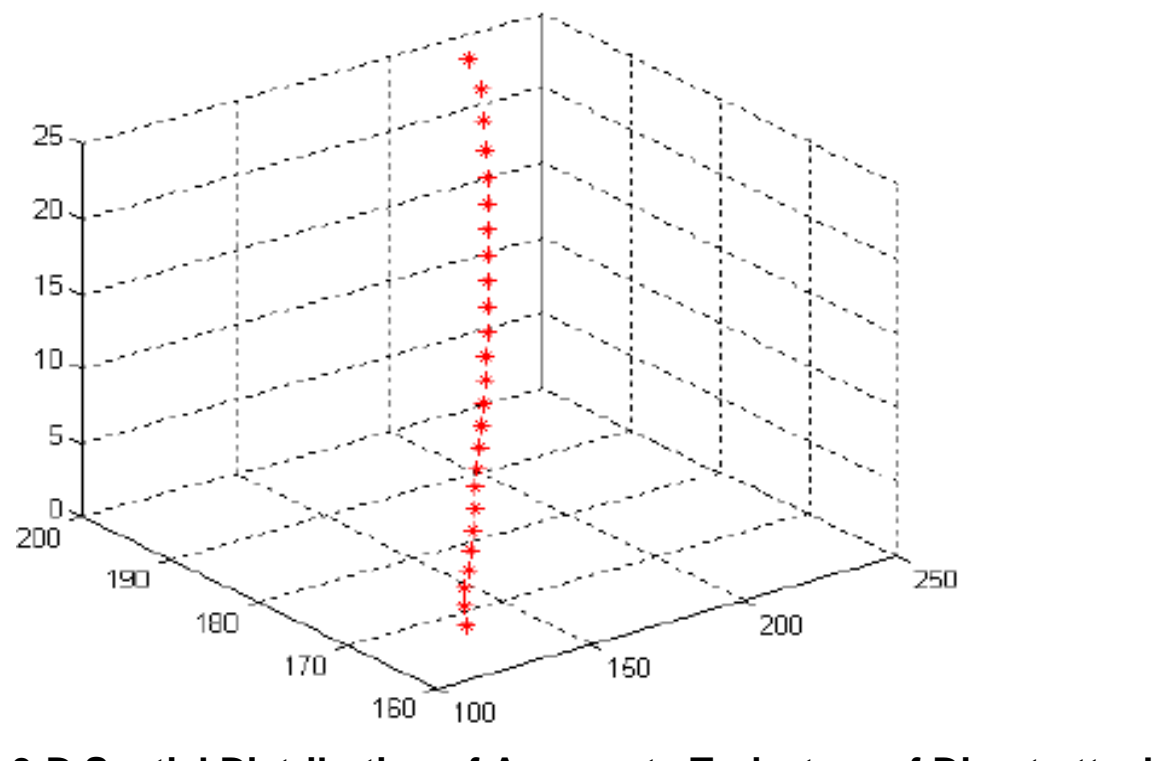

Figure 2. 3-D Spatial Distribution of Aggregate Trajectory ol Direct-attack

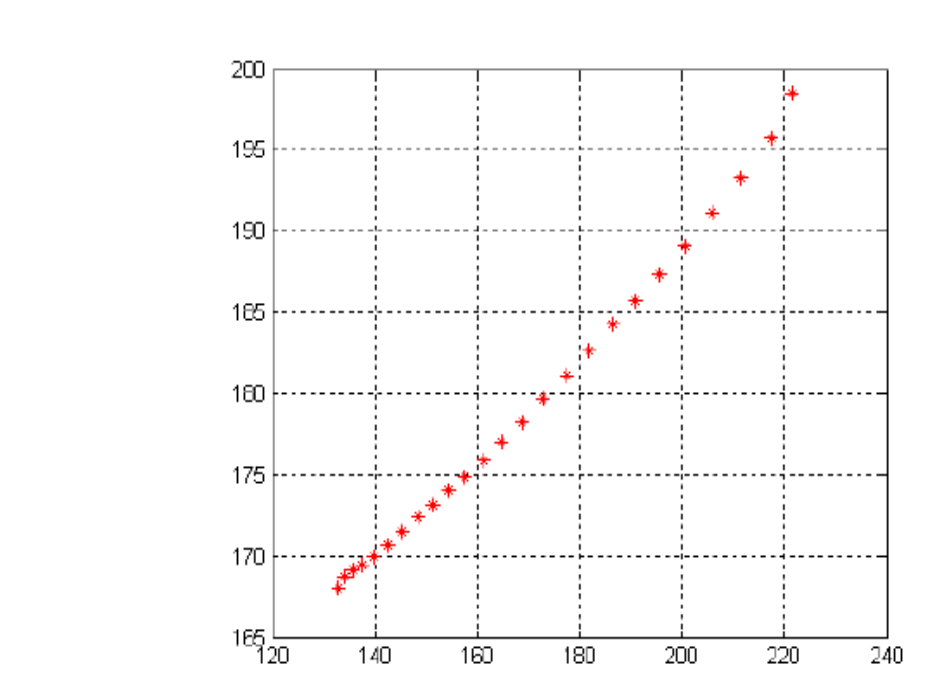

Figure 3. 2-D Spatial Distribution of Aggregate Trajectory of Direct-attack 


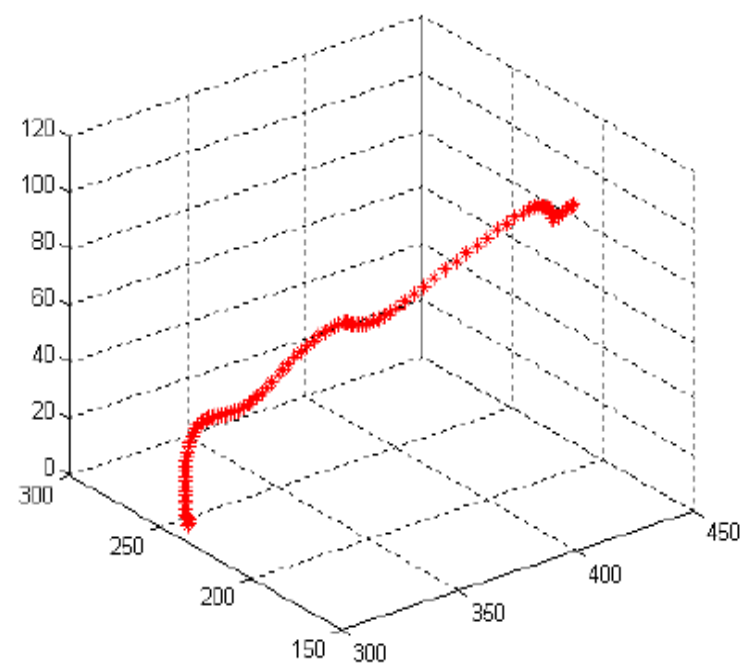

Figure 4. 3-D Spatial Distribution of Aggregate Trajectory of Dribblingattack

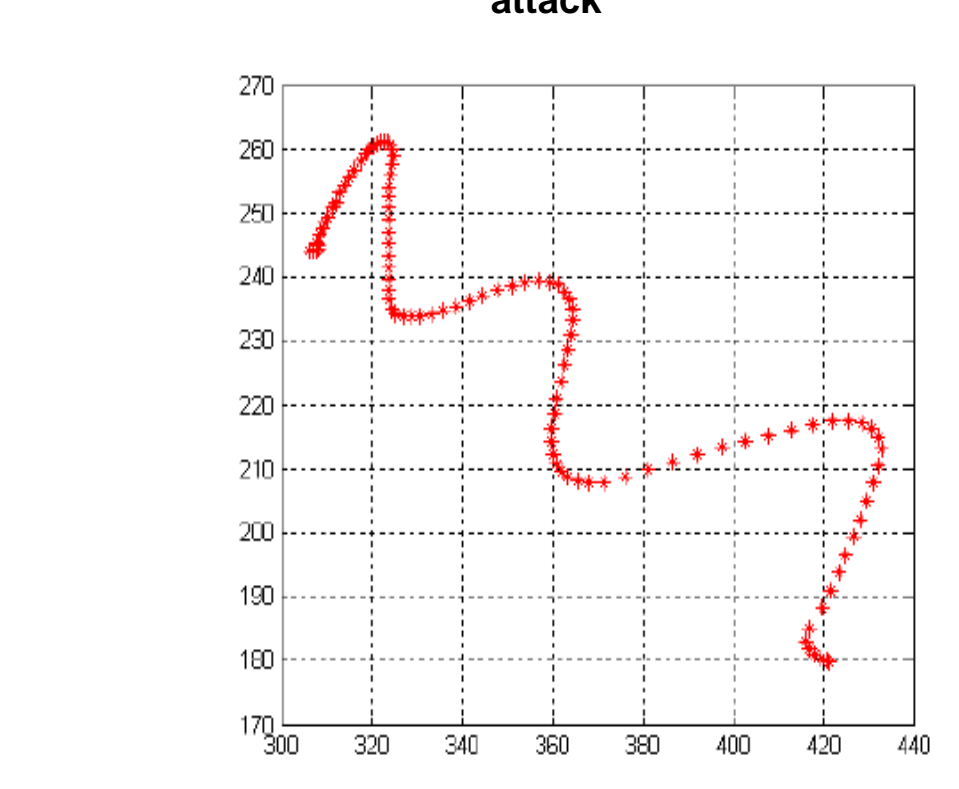

Figure 5.D Spatial Distribution of Aggregate Trajectory of Dribbling$\checkmark$ attack

Given the identified interactive trajectory of personal attack event $A T=\left\{s_{1}, \ldots, s_{n}\right\}$, using the average cumulative error testing to determine its spatial distribution is similar to the direct distribution. Set the two assumptions:

$$
\left\{\begin{array}{l}
H_{0}: f(X, Y \mid k, c)=0 \\
H_{1}: f(X, Y \mid k, c) \neq 0
\end{array}\right.
$$

Which, $X=\left\{x_{1}, \ldots, x_{n}\right\}$ and $Y=\left\{y_{1}, \ldots, y_{n}\right\}$ are each of the projection component of trajectory in $X$ and $Y$ directions. $s_{i}=\left(x_{i}, y_{i}\right) . k$ and $c$ are linear distributed parameter of estimation. Using the least square method can estimate function expression $y=L(x \mid k, c)$ of 
the linear distribution. Based on this, you can calculate $A T$ the average cumulative error $A E$ :

$$
A E=\frac{1}{n} \sum_{i=1}^{n}\left[y_{i}-L\left(x_{i} \mid k, c\right)\right]
$$

According to the formula(9), the smaller value of $A E$ indicates that $A T$ distribution is close to a straight line. Thus, you can fine classification standard definition of personal attack

$$
P_{\text {fine }_{-i}}(G)= \begin{cases}\text { Direct attack } & A E \leq \text { thres } \\ \text { Dribbling attack } & A E>\text { thres }\end{cases}
$$

Which, thres is threshold parameter of predefined.

\section{Experimental Analysis And Results}

In order to verify the proposed tactics mode recognition method, using the 2012 FIFA World Cup (World Cup 2012) as the test data of video games. All games videorare directly transcription from the TV live broadcast. All 64 matches of the video is stored in MPEG-2 compression format, video frame size is $704 \times 576$.

\subsection{Acquisition of True Values of Experimental Data for Semantic Event and Tactical Mode}

Preparations of true values of experimental data are Cmportant to objectivity and effectiveness of the method's evaluation results. In the sports videos, the definition of both event's semantic type and tactical modesare subjective to tertain extent. For the same video script, the identification of the semantic concept of the belonged event type is often different from one another for different observers. The labeling of tactical mode is also of subjectivity. To obtain true values of the semantic category and tactical mode of competitive event more objectively and accurately, we invited five football professional persons to annotate semantic classes (whether it's attacleyent) and Iactical mode (coarse interactive or fine interactive mode) for all segments $n$ all related videos. They have years of experience in football game or being as coach. They gained accurate understanding and mastery of the occurrence, transformation and ending of attack model in games. So their labeling results are more credible and impartial. For labeling, there're two principles to obey:

Visual fragments to be marked can be made as long as possible to ensure the completeness of attack event.

To mark as many events as possible as to guarantee the variety of tactical modes in the attack event. In order to ensure the diversity in the event of tactical offensive.

Event fragment semantic categories and tactical mode of true value are confirmed based on annotations made by 5 professionals with voting mechanism. Finally we have 1235 fractions of attack events for 64-round competition videos, as seen in Table 2. Different interactive tactica modes involved are listed in Table 3.

Duning the performance testing, we adopted the algorithm in [15] to perform automatic detection of attacks. That method is proposed on multi-mode fusion analysis idea, detecting from networking and broadcasting words when one attack even happens with the help of predefined event text keywords. Then according to time detection of broadcast sport game video timing board, it utilizes limited state machine model to determine the boundary where the event in videos starts and ends to complete the detection. The method is obviously superior over other similar methods in terms of detection accuracy. 
Table 2. Attack Events for Tactic Mode Recognition

\begin{tabular}{|c|c|}
\hline Event categories & The number of events \\
\hline Goals & 168 \\
\hline Shooting & 413 \\
\hline Corner & 125 \\
\hline Free Kick & 529 \\
\hline ALL & 1235 \\
\hline
\end{tabular}

Table 3. Experimental Data for Tactic Interaction Mode Recognition

\begin{tabular}{|c|c|c|}
\hline \multicolumn{2}{|c|}{ Interactive mode } & Mode number \\
\hline \multirow{2}{*}{ Coordinated attack } & Interception attack & 134 \\
\cline { 2 - 3 } & No interception attack & 662 \\
\hline \multirow{2}{*}{ Personal attack } & Direct attack & 311 \\
\cline { 2 - 3 } & Dribbling-attack & 128 \\
\hline
\end{tabular}

\subsection{Experimental Result of Interactive Mode Recognition}

To make quantitative assessment of the algorithm's performance, we set two indexes like recall $(R)$ and precision $(P)$ ratio of the mode recognition. They are respectively defined as follows:

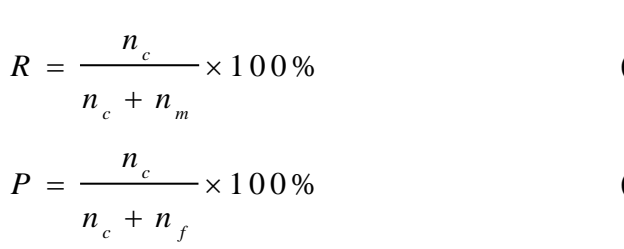

where, for any mode, $n_{c}$ refers to the number of attack events identified correctly; $n_{m}$ means the number of events which were not recognized; $n_{f}$ is the number of goal events where it was falsely determined as the interactive mode. Interactive recognition algorithm's effectiveness test was completed on all 1235 attack event fragment. Results are shown in Table 4 and 5.

\section{Table 4. ExperimentaResults of Coarse Interaction Mode Recognition}

\begin{tabular}{|c|c|c|c|}
\hline Mode & Mode number & Recall (\%) & Precision $(\%)$ \\
\hline Coordinated attack & 796 & 89.4 & 93.2 \\
\hline Personal attack & 439 & 88.2 & 82.2 \\
\hline \multicolumn{4}{|c|}{ able 5 Experimental results of fine interaction mode recognition } \\
\hline Mode $=$ & Mode number & Recall (\%) & Precision $(\%)$ \\
\hline Intercep ion attack & 134 & 74.6 & 81.3 \\
\hline No interception attack & 662 & 88.1 & 89.1 \\
\hline DDrect attack & 311 & 83.9 & 86.4 \\
\hline Dribbling-attack & 128 & 85.2 & 70.3 \\
\hline
\end{tabular}

From Table 4 and Table 5, we can learn that the proposed tactical mode recognition method makes desirable results. We think two factors can have effects on its performance of identification:

Robustness of multiple object detection and trajectory algorithm. The trail of players and the ball is one of the objects to establish medium expression of tactics in attack event video fractions. The accuracy of trajectory information to be obtained is associated directly with the 
superior or inferior performance of recognition. In some cases, occlusions are seriously seen among players and players with the ball so that manual methods can't determine the affiliation relationship between right trace and object. That's why differences and errors exist in multi-object trajectory information;

Accumulative errors in the calculation of inlaid trajectory. To estimate the inlaid tacks we used global motional estimation method to eliminate camera movements in the broadcast videos. To estimate the global movement estimation transformation matrix is the process of being optimized. The transformation of each frame has errors. Along with evolution of time series, errors will accumulate and aggrandize. Hence, resulted mosaic trajectory s can't reflect correctly the sport nature of players and the ball. Based on the status quo of sports video game tactical analysis method. This paper presents tactical pattern recognition method is the first analysis method based on broadcast video Football Tactics. On the other hand, in the ball team project. The players and the ball are essential to the game participants, is an important movement object in venue. Trajectory can reflect play and Implementation of various leam tactics and personal tactical skills in the process of game.

\section{Conclusion}

Tactical analysis aims to find players in sports between personal and team to use tactical mode or competition strategy in finish the task in the process. Based on application of automatic analysis method of video games tactic, not only can meet sports professionals to obtain competition strategy needs, but also the najority of sports fans can browse to the game content. Trajectory information team is an important feature of revealing the tactics of the situation. According to the trajectory of the ball, can complete the tactical interaction pattern recognition and classification in the game. Therefore, propose the player and the ball trajectory analysis to produce tacticat forms of expression the trajectory team projects equally applicable to other tactical analysi applications based on in multiple objects (player, ball).

\section{References}

[1] Z. Yingying and Z.^Yahyan "Sports villeo type logo bag model of lens and word based classification", Journal of Computer Aided Design \&amp, computer graphics, vol. 09, (2013), pp. 1375-1383.

[2] Z. Like, "On the constuction of sperts multimedia video library", Electronic test, vol. 18, (2013), pp. 212213.

[3] C. Wenhua, Z. Shan, S. Jun and W. deeply, "Media fission and fusion Sina digital marketing evolution", Successful marketing, vol 12, (2013), pp. 39-49.

[4] B. Jiang, Z. Bin and LO Dongsheng, "A robust field ball video goal detection method", Journal of National University of Defense Technology, vol. 05, (2013), pp. 85-90.

[5] X. Libin, "Advance in research on players' technical and Tactical Features in pattern recognition and neural mechanism", Journa of Nanjing Sport Institute (NATURAL SCIENCE EDITION), vol. 05, (2013), pp. 4755.

[6] W. Yunsheng, "A preliminary study on the application of computer in tennis training", Electronic test, vol. 24, (2013), pp. 204-205.

[7] T. Taki, J. Hasegawa and T. Fukumura, "Development of Motion Analysis System for Quantitative Evaluation of Teamwork in Socer Games", IEEE International Conference on Image Processing. Lausanne: IEEE, vol. 3, (1996), pp. 815-818.

[8] S. Hirano and S. Tsumoto, "Finding Interesting Pass Patterns from Soccer Game Records", European Conference on Principles and Practice of Knowledge Discovery in Database. Pisa: Springer, vol. 3202, (2004), pp. 209-218.

[9] C. Kang, J. Hwang and K. Li, "Trajectory Analysis for Soccer Players", IEEE International Conference on Data Mining Workshops. Hong Kong: IEEE, (2006), pp. 377-381.

[10] D. Zhong and S. Chang, "Structure Analysis of Sports Video Using Domain Models", IEEE International Conference on Multimedia \& Expo. Tokyo: IEEE, (2001), pp. 713-716.

[11] T. Orazio, C. Guaragnella, M. Leo, et al., "A New Algorithm for Ball Recognition Using Circle Hough Transform and Neural Classifier”, Pattern Recognition, vol. 37, no. 3, (2004), pp. 393-408. 
[12] X. Yu, Q. Tian and K. Wan, “A Novel Ball Detection Framework for Real Soccer Video”, IEEE International Conference on Multimedia \& Expo. Baltimore: IEEE, vol. 2, (2003), pp. 265-268.

[13] D. Liang, Y. Liu, Q. Huang, et al., "A Scheme for Ball Detection and Tracking in Broadcast Soccer Video", IEEE Pacific-Rim Conference on Multimedia, Jeju Island: Springer, (2005), pp. 864-875.

[14] F. Dufaux and J. Konrad, "Efficient, Robust, and Fast Global Motion Estimation for Video Coding", IEEE Trans. on Imapge Processing, vol. 9, no. 3, (2000), pp. 497-501.

[15] C. Xu, J. Wang, K. Wan, et al., "Live Sports Event Detection Based on Broadcast Video and Web-casting Text”, ACM International Conference on Multimedia, Santa Barbara: ACM, (2006), pp. 221-230.

\section{Author}

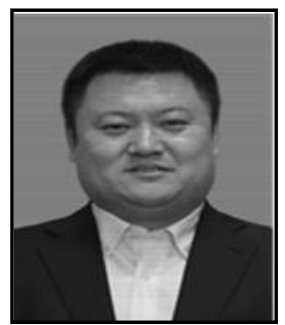

Liu Gang, he is an Associate Professor at Physical Education Department of Harbin Engineering University. He is in the research of physical education and training

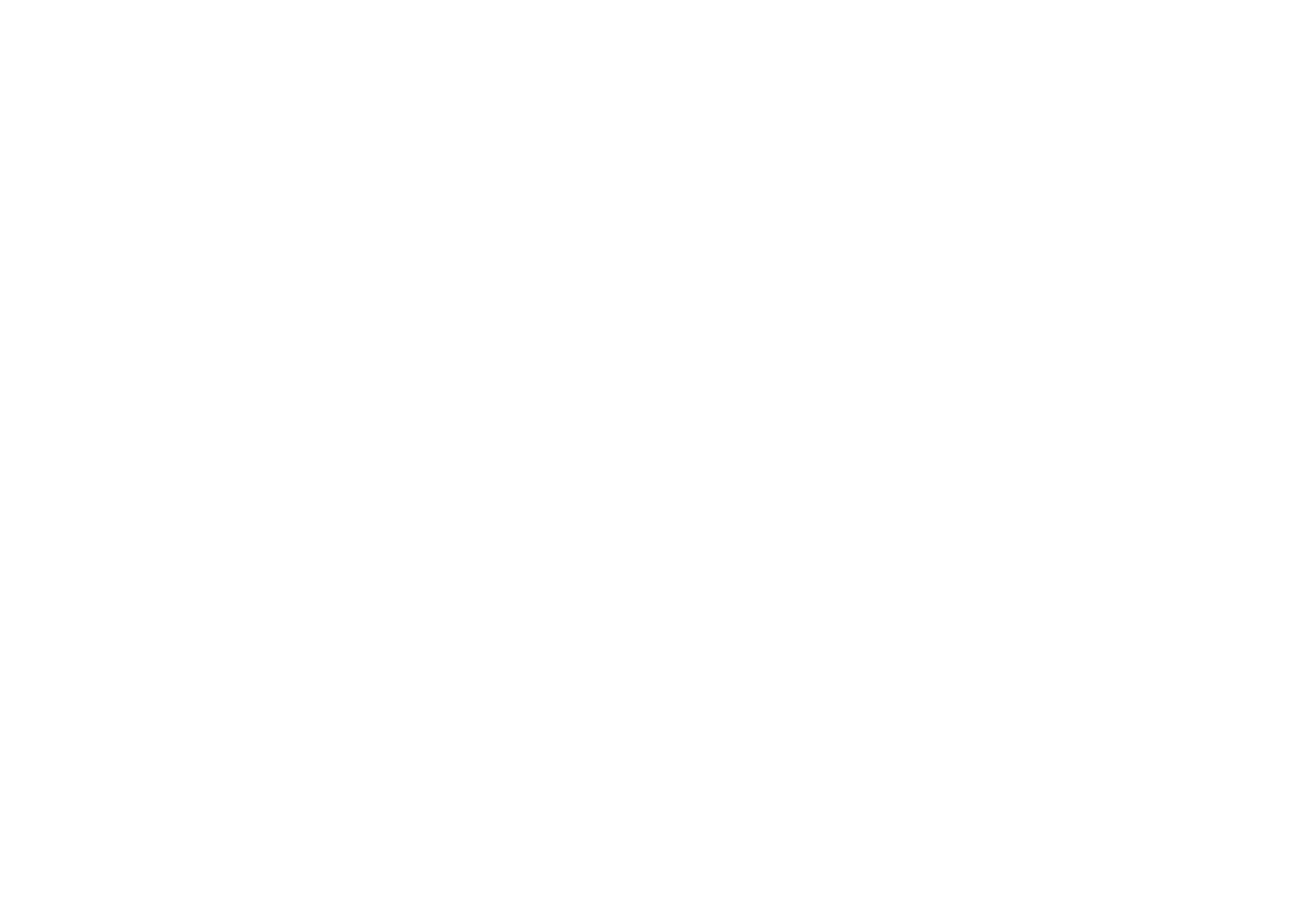


International Journal of Multimedia and Ubiquitous Engineering Vol. 9, No. 12 (2014)

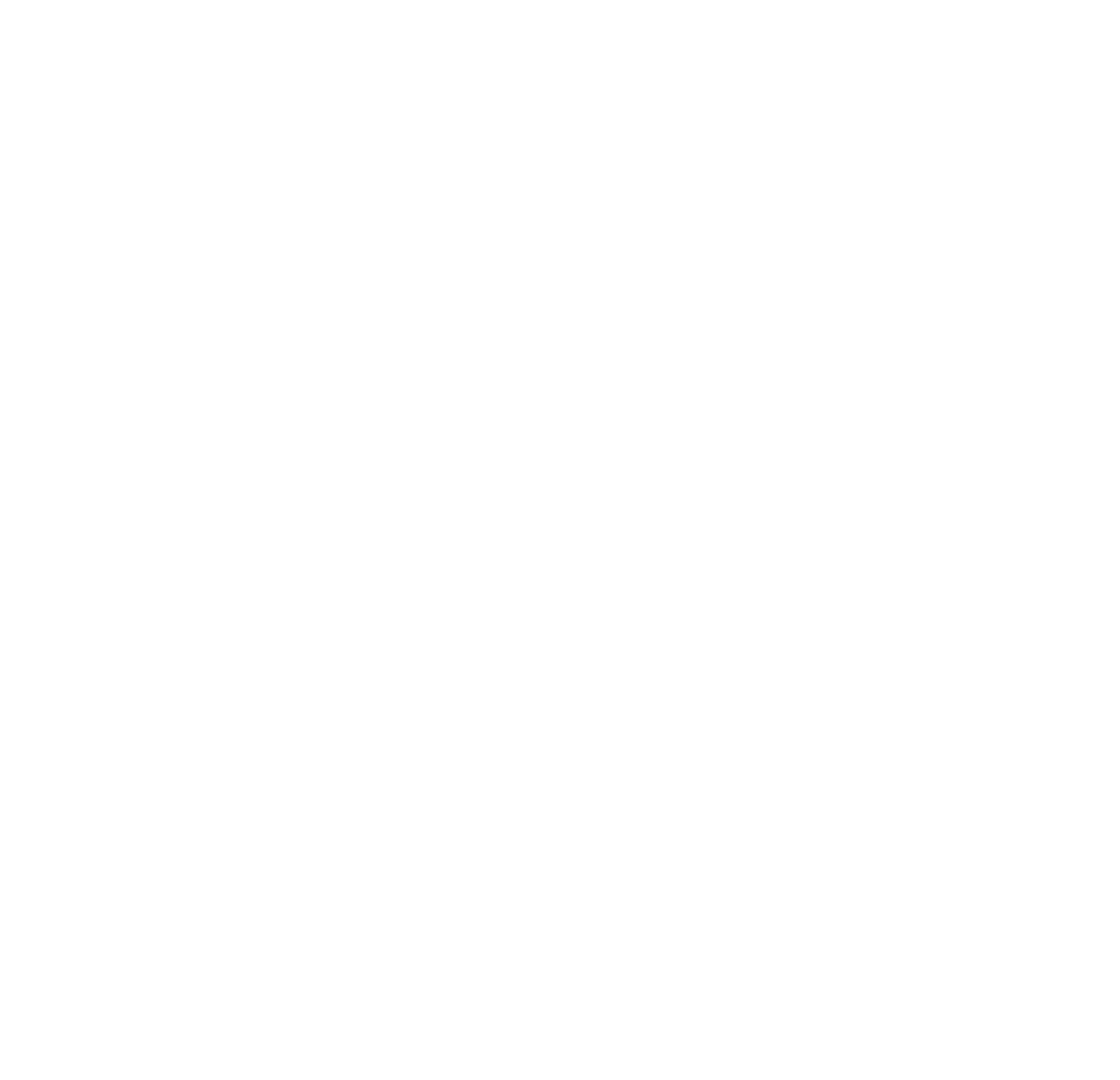

